any further attempts at insurance by all the means in my power. His confidence in me is indeed a poor thing if it will not survive one contrary view, and in such a circumstance the sooner he sought another doctor the better, but repeated opinions adverse to mine would be bound to bring about my downfall. That in itself does not matter, but, remember, it was to benefit the patient that the truth was withheld, and now through repeated unsuccessful attempts at insurance, the mask is off, and he sees himself as an unfit man.

The attitude of Insurance Companies to private doctors is often misunderstood. They are, as a rule, anxious to share any medical information that may have come their way. If a patient goes to his doctor saying he has been rated up or declined for life assurance, and the doctor does not know why, he is at perfect liberty to write to the company, who will willingly give him the reason, usually through their own principal physician; but they will certainly refuse to tell the proposer himself. In this refusal they recognise that it is for the doctor to decide whether much or little shall be made of what has been found. That will depend on many things, the patient's temperament, occupation, family circumstances, and so on, that are known only to the private doctor, but above all it will depend on whether or not any treatment will help.

Usually people only seek their doctor's advice when they feel ill. They seldom go for overhauls, pure and simple, so it often happens that a company tumbles on some symptomless condition that could only be found by routine examination-a proceeding to which the public are singularly disinclined to submit themselves, if they can possibly help it. Sometimes it is this trivial matter of a medical examination that prevents a man from making very necessary provision for his wife and family. Is it fear, or what? or is it just because he can't be bothered?

\title{
THE EXAMINATION FOR LIFE ASSURANCE
}

\author{
By D. R. CRABB, M.B., B.S. \\ (C.M.O., Atlas Assurance Co., Ltd.)
}

\section{Introduction.}

The medical examination of a candidate for life assurance may be carried out by the company's chief medical officer at its head office, or by a practitioner nominated for that purpose by the insurance office. The former will see many cases during the year and will devote all or a large part of his time to life assurance work, whereas such work will only form a small part of the general practitioner's daily task. Unless there is some special reason for asking the candidate to attend at the chief office for examination, the choice of examiner is generally dictated by the convenience of the candidate. For reasons set out in this paper, it is beholden to the practitioner to examine all such cases with care and circumspection.

\section{The Form of Report.}

The form on which the report is made varies with different offices. The printed form, however, is merely intended as a framework on which to build the report, and any replies should be amplified if, by so doing, a clearer picture is obtained. Definite answers should be given; if the answer is merely yes or no, it should be written so, and not indicated by a tick or stroke which may mean anything. What is wanted in the report is a clear picture of the person's family history, past history, present condition, build and temperament, together with a careful account of any deviations from normality, even though such deviations do not appear to be affecting the health. As an example, consider the question of underweight and overweight; such a person may appear in perfect health at the examination, yet it is known that this overweight is an increasing liability with advancing years, and that there is an increased risk of an earlier death as compared with those of normal weight. Similarly, with the tall, underweight adolescent, there is a greater risk of developing tuberculosis than in those people of a normal build. Thus, in these cases, although they may be in good health, there is an added risk from 
an assurance point of view. This added risk may be increased or diminished by a consideration of the family and personal past history.

\section{The Examination.}

This will fall into three parts:-

I. The family history.

2. The personal history.

3. The clinical examination of the patient, including the examination of the urine.

\section{The Family History.}

On the proposal form, which the doctor will have before him, the proposer will have set out details of his family history. These details will consist of age of parents and siblings, their state of health, if alive, and the age at death and the cause of death, if deceased.

Not infrequently, the cause of death in any case may be given in vague terms, and if such is the case the answers should be amplified. Several expressions which may occur and require amplification are:-

(a) Nervous breakdown.

This may be found both in the family history and in the personal history, and may mean anything from insanity to a temporary exhaustion following a period of anxiety or overwork. The nature of the "nervous breakdown" should always be elicited.

\section{(b) Respiratory conditions.}

Respiratory conditions such as bronchitis, pneumonia, influenza, pleurisy and asthma may represent a terminal complication of some other disease, or may have been tuberculous in nature. Enquiry should be made as to the length of illness prior to death, and as to any other serious illness that the relative may have had, whether sanatorium treatment had even been necessary.

\section{(c) Childbirth.}

This may be given as the cause of death, when in point of fact it may have been some other disease which, complicated by childbirth, had resulted in death.

(d) Cancer.

Where there is a family history of cancer, enquiry should be made as to the site of the cancer, especially if there is more than one such case in the family.

(e) Peptic ulcer.

Enquiry should be made as to whether any other members of the family have ever suffered from a similar condition.

\section{(f) Heart disease.}

The nature of the disease should be discovered if possible.

\section{(g) Tuberculosis.}

Most offices have a specific question as to whether any near relatives have suffered from or died from pulmonary tuberculosis. It is not sufficient to give the bare details. The office really wants to know whether there was contact between the proposer and the tuberculous person, and if so, of what degree and duration, and how long ago it happened.

\section{Personal History.}

The proposer may have stated in his proposal that he has had no serious illnesses. Further enquiry by the examiner may reveal that this is not really the case; the illness may have been temporarily forgotten, or else considered insignificant. 
Many reports carry the phrase "Usual childish ailments." It is better to state the nature of the ailments; some may be of significance, such as whooping cough and measles, which may leave behind a chronic cough eventually leading to the development of chronic bronchitis or bronchiectasis. Scarlet fever may have damaged the kidney; tonsillitis (sic), the heart, etc.

Where there has been any illness, the details of that illness should be given, where such details may be of value.

"Pleurisy-r936" (supposing such to be the case), is not really sufficient; was the pleurisy dry or was there an effusion?; how long was the patient ill?; what treatment was adopted?; was the sputum examined, and if so with what result?; was the chest X-rayed at the time, or subsequently, and if so, where, with what result, and what was the date of the last of such examinations.

"Indigestion, dyspepsia," are other phrases which often occur in the personal history and require further details as to their nature-if considered to be due to a trivial dietitic upset, say so-if not, give further details. Accidents should be amplified as to the nature and course thereof.

\section{Venereal disease.}

If admitted, details should also be given of the date and duration and nature of treatment, and in the case of syphilis, the date and result of the Wassermann or Kahn reaction.

\section{Personal habits.}

The amount of alcohol consumed should be ascertained and details of the usual daily consumption given. Details of tobacco consumption should also be given if excessive.

The amount of exercise taken should be ascertained; does the proposer lead an active life or a sedentary life?; does he take any special exercises, such as outdoor games, gardening, etc.

\section{THE EXAMINATION OF THE PATIENT}

The examination of a patient for life assurance purposes differs but little from the examination of a patient who comes for advice on account of illness, except that in the case of life assurance examination there are as"a rule no presenting symptoms to lead one along any particular line of approach, unless there should be any special features in the family history or the personal history which require particular attention.

To cover all the points which have to be borne in mind, it would be necessary to write a book on physical diagnosis and clinical medicine, so it is intended to limit one's remarks in this paper to certain points which are important from the point of view of life assurance.

The actual order of examination may vary with the individual examiner, but it is important that a definite routine of examination should be adopted in order to avoid overlooking any point.

The candidate for life assurance examination frequently arrives at the consulting room in an apprehensive state of mind, and one should do as much as possible to reassure him by a friendly manner whilst obtaining the family and personal history.

\section{General Appearance.}

Here one should note the race and general appearance and manner, whether robust or weedy, tired or nervous; also pallor or cyanosis, and whether there is any dyspnœa. Any peculiarity of gait will probably have been observed when the patient enters the room. Exopthalmos, ptosis and tremor of the face will also be evident if present. The apparent age should also be noted-whether apparently older or younger than the stated age.

\section{The Essential Measurements.}

The height and weight of the individual, together with circumference of chest expanded and contracted, and the abdominal girth are almost invariably asked for, and they should be measured and not estimated.

(a) Height and Weight.

It is as well to state whether the height and weight are taken with the patient clothed or stripped, as the statistical tables of body build used by the company are as a rule based on averages of clothed weight. 
(b) Chest Measurements.

These should be taken at the level of the nipples. The arms should be raised from the sides and the tape measure passed round the chest at the level of the inferior angle of the scapula posteriorly and the nipples anteriorly. The arms should then be lowered to the sides and the measurements carried out. The actual measurement should be repeated two or three times as the range of expansion improves with practice. A greater degree of emptying of the chest can often be obtained by asking the patient to whistle as he exhales, and to keep whistling as long as possible. Care should be taken to see that the measurements are those of expansion and contraction of the chest, and not of extra movement produced by bracing the shoulders upwards and backwards.

In the case of women the tape measure should be passed under the breasts at the junction of the lower border of the breast tissue with the chest wall, and not over the nipples. In this way a more accurate measurement of chest movement will be obtained, and any fallacy due to compression of breast tissue by the tape measure will be avoided.

\section{(c) Abdominal Girth.}

The measurement of the abdominal circumference is made in a horizontal plane at the level of the umbilicus, the patient standing with the abdominal muscles relaxed and with the chest in a position of easy expiration.

\section{The Respiratory System.}

Cyanosis and dyspnœa will already have been observed if present. The nose and throat should be examined for any evidence of chronic sepsis or obstruction. Particular attention should be paid to the tonsils, any scarring or pocketing as a result of old infection being noted. The neck should be examined for enlarged glands, particularly in the tonsillar and supraclavicular regions, the size and shape of the thyroid gland, and the trachea examined for deviation by palpation in the suprasternal notch. Any clubbing of the fingers should be noted also at this stage.

The patient should be stripped to the waist and the chest observed in a good light. This applies to women as well as men. There is no place for false modesty in the consulting room, and it is impossible to detect diminution of movement and wasting or flattening of the chest? wall with the chest covered.

The shape of the chest, whether long and narrow or round, and any inequality of movement between the two sides of the chest wall should be looked for, and also any marked flattening or wasting, especially in the infraclavicular regions. At the same time, any dilated veins on the chest or neck will be remarked and described.

The lung fields are then examined in the usual way by palpation, percussion and auscultation, and any abnormalities found entered in the report.

It is important to be on the look out for evidence of early tuberculosis, slight bronchiectasis, chronic bronchitis, and in older subjects, for early carcinoma of the lung. The signs may be minimal, and the earliest sign may be a slightly decreased movement with some flattening on the affected sign. Recent experience has shown that early pulmonary disease may be present, especially tubercle and neoplasm; with very little evidence present in the way of physical signs. If there is the slightest doubt in the examiner's mind as to the healthy. condition of the chest, he should recommend that an X-ray of the chest be obtained before a decision is given. If an early case of tuberculosis or neoplasm is missed, the loss to the office is likely to be heavy, for the loss will probably occur before the policy has been long in force.

\section{The Cardio-Vascular System.}

The examination of the cardio-vascular system is directed towards an assessment of the functional efficiency of the heart and blood vessels, and the possible future detrimental effect of any abnormality which may be detected. Should any abnormality be detected, one's findings should be given in full. .For example, in answer to the question "Are the heart sounds normal?" it is not really sufficient to write down "Systolic murmur at apex," as I have not infrequently seen. One wants to know whether it is conducted, and if so, where; where it is heard with maximum intensity; whether it is heard equally well with the patient standing up and lying 
down; what is the effect of exercise, $(a)$ on the murmur, $(b)$ on the pulse rate; what is the increase in rate, and how long it takes to return to normal after exercise; does moderate exercise cause dyspnœa; is a thrill palpable, and if so, where; is there engorgement of the veins of the neck; is the liver enlarged, and is there any œdema. Give all the information with regard to the abnormality that can be obtained by clinical examination.

The main points which are expected are:

\section{The pulse rate.}

The character of the pulse wave, the rhythm, the tension, and the state of the artery wall should all be reviewed. Any abnormality should be amplified. Thus, if an irregularity of the pulse is found, its significance should be stated. If considered of no significance, the reasons for considering it so should be given. In other words, a complete diagnosis should be attempted. A tachycardia may be due to nervousness; if present, a further count should be taken later in the examination, by which time the patient will probably have settled down. If the tachycardia persists, every endeavour should be made to ascertain the cause.

\section{The position of the apex beat.}

This should be described in terms of the intercostal space in which it is palpable, and its distance as measured from the mid-sternal line.

\section{The size of the heart.}

This can be estimated very roughly by percussion of the area of deep cardiac dullness and the position and character of the apex beat. The estimation is not particularly accurate, and more important is the estimation of the condition of the myocardium, as shown by its response to exercise, and the presence or absence of any confirmatory signs of a failing myocardium, such as venous engorgement in the neck, enlargement of the liver, congestion of the pulmonary bases, œdema of the dependent parts of the body, dyspnœa, at rest or otherwise.

\section{The character of the heart sounds.}

As previously mentioned, any abnormality, especially any murmurs which may be heard, should be described in full and their significance assessed.

\section{The blood pressure.}

In many cases the assurance office will send a leaflet describing the method to be adopted in estimating the blood pressure. The chief differences lie in the methods used for estimating the diastolic pressure. Whilst the majority of offices adopt the estimation of the diastolic pressure as being at that point at which there is a sudden transition in the sound from the loud beat to a soft murmur, others still adopt the estimation of diastolic pressure as being at that point at which all sounds disappear. (This latter method has been disapproved by the British Blood Pressure Committee set up by the Cardiac Society of Great Britain and Ireland, I938.-ED.)

If a definite method is indicated by the office concerned, that method should be adopted, as their statistical tables, and hence their rating for any impairment, will be based on pressures estimated in that way.

If no method is indicated, the diastolic pressure is estimated as being at that point at which the sudden transition in the intensity of the beat occurs.

In general, the systolic pressure is best estimated by the palpatory method, whilst the diastolic is estimated by the auscultatory method.

Should the pressure readings be above normal, the patient should be made to lie down and relax, and further estimations made. A high reading should always call for further estimations, and if it remains high, one should state in the report that this figure represents the best of repeated readings.

Any abnormality should call for comment, particularly any abnormality in the diastolic pressure. In general, a blood pressure of $140 / 90$ is considered as being the borderline between the standard and the substandard life. A raised diastolic pressure is found to be of more 
significance in a younger patient, whereas over fifty, the systolic is the more significant. In this, one is of course generalising, the $140 / 90$ figure being relaxed somewhat in the over fifties.

\section{The Nervous System.}

One is always asked to state the condition of the knee jerks and the reaction of the pupils to light. As a rule, there is a further question which asks, "Is there any evidence of disease of the nervous system?" or words to that effect.

As a rule, one should test the movements of the eyes, note the presence of any tremors of the eyelids, lips, tongue, or fingers, the presence of any wasting or paresis, and the test for ataxia.

Obviously, should any abnormality be detected, the examination of the nervous system will be gone into in more detail in order to discover the cause of the abnormality.

\section{The Alimentary System and the Abdomen.}

The mouth should be examined to ascertain the condition of the tongue and teeth, particularly as to whether the latter form an efficient masticatory unit, and as to the presence of sepsis at the gum margins.

The abdomen should be examined with the patient lying down, in a good light, and with the abdominal muscles relaxed. In the case of a woman, she should be asked to remove any corsets or abdominal belt before the abdomen is examined.

By palpation, the presence of any abnormal rigidity or tenderness will be discovered and commented on, as well as any enlargement of liver, spleen, or kidneys, and the presence of any abnormal swelling or tumour. Should any abnormality be discovered, its characters should be described in detail.

Any scars on the abdominal wall should be described.

The existence of a hernia will best be discovered with the patient in a standing position.

The anus should be examined for the presence of piles, fistula, or fissure. If any abnor mality is discovered, and in the case of persons over the age of forty-five, it is as well to do as rectal examination. A carcinoma of the rectum or enlargement of the prostrate may be misseds unless this is done.

\section{The Genito-Urinary System.}

In the male, the presence of scars from old syphilitic disease should be looked for, as well as the presence of varicocele, hydrocele, enlargement of the testes, or thickening of the vas deferens.

In the female, the examination of the reproductive system should be compared to the history and to the abdominal examination. Enquiry should be made as to the character and regularity of menstruation, the presence of intermenstrual discharge, its character and duration; whether pregnancy has occurred, and if so, how many times, when, and with what result; the character of the labour, if children have been born, and was it uneventful or complicated. These enquiries should be made whether the woman is married or not, though it should be done as tactfully as possible. In general, the most tactful way is to put the questions directly, in a matter of fact way, in the same way as all other questions.

Should the examiner, as the result of his questioning and his examination of the abdomen, suspect the presence of pelvic disease, he should recommend that a gynæcological examination should be made. It is not, as a rule, necessary for the examiner to make a pelvic examination.

\section{The Urine.}

A specimen of urine should be obtained, and this should be passed in the presence of the examiner, in order to avoid the possibility of substitution. This, of course, is not practical in the case of women, and they can be asked to pass the specimen whilst in the premises. If this is not possible, the examiner must be content with a specimen which the patient brings with her.

The urine should be examined as a routine, for specific gravity, reaction, albumin and sugar, and its appearance noted. If there are parts in the history or examination which suggest such further tests, it should be examined for blood, pus and bile. 
Further pathological examination of the urine is not asked for, but if any abnormality is detected, the examiner may advise the company that such further examination should be done.

Sugar.

Either Fehling's or Benedict's test may be used. Benedict's is preferable, and is specified by some companies as it is less open to fallacies.

Benedict's Test.

Five c.c.s of the solution should be measured into a boiling tube. (As a practical point it is worth while using a large boiling tube of heat-resisting glass; it can be placed directly in the flame without a preliminary warming up, and being large, it is less likely to boil over, and in the long run it is more economical, as tubes are not cracked by heating too rapidly).

The reagent should be brought to boiling point and then eight drops of urine added, and the mixture boiled for a further two minutes. It is as well to time the two minutes as sugar may be missed if reduction is not complete-and it always seems a very long time when holding a test tube over the flame! The solution is then allowed to cool, and the colour of any deposit is noted.

Should a reducing substance be detected, the history and clinical examination should be re-checked to see if there are any confirmatory signs or symptoms of diabetes.

In all cases in which reduction of Benedict's solution occurs, the company should be advised to have a glucose tolerance test before accepting the case.

\section{Albuminuria.}

The two tests commonly in use for the detection of albumin in the urine are:-

I. Boiling, and the use of diluted acetic acid.

2. Heller's concentrated nitric acid test.

If albumin is detected in the urine of a young person, it may be a functional or orthostatic albuminuria. For this reason, should albumin be discovered in such a person it will save a great deal of time and correspondence if the patient is asked to bring a specimen of urine passed immediately on waking in the morning. This should be examined and the findings included in the report before sending it to the head office.

If albuminuria is persistent, a pathological examination of the urine will almost always be asked for.

\section{Specific gravity.}

The urine should be allowed to cool before estimating the specific gravity as this will give a higher reading than if it is taken whilst the urine is still warm.

The healthy kidney should be able to secrete urine of a specific gravity of Iozo or higher. If at examination the specific gravity is found to be low, it may well be due to cold, nervousness, or having recently imbibed a large amount of fluid. If any doubt exists it is worth while asking for a further specimen of urine passed first thing on waking, the patient having taken no fluid since teatime the previous day. If the specific gravity remains low, further investigation should be advised.

\section{Conclusion.}

It will be seen that the examination for life assurance calls for a careful and thorough clinical examination-nothing more. A few final points are worth bearing in mind. The writing should be legible and the report should be read through after completion to ensure that no questions have been left unanswered and that there are no obvious inconsistencies or mistakes. Lastly, the conclusion of the examining doctor should NEVER be communicated directly to the candidate or even hinted at. The examiner is making a confidential report to the assurance office and it must remain confidential. 